\title{
Rapid mixing of colliding picoliter liquid droplets delivered through- space from piezoelectric-actuated pipettes characterized by time-resolved fluorescence monitoring
}

Cite as: Rev. Sci. Instrum. 90, 055109 (2019); https://doi.org/10.1063/1.5050270

Submitted: 29 July 2018 . Accepted: 27 April 2019 . Published Online: 21 May 2019

Jamie L. Y. Wu (巫柳依) (D), Friedjof Tellkamp (D), Mazdak Khajehpour (D), Wesley D. Robertson (D), and R. J. Dwayne Miller (iD)
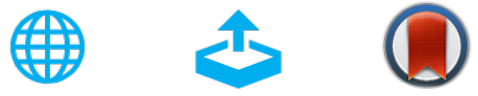

View Online

Export Citation

\section{ARTICLES YOU MAY BE INTERESTED IN}

Raman spectrometer for the automated scan of large painted surfaces

Review of Scientific Instruments 90, 053101 (2019); https://doi.org/10.1063/1.5088039

Femtosecond time-resolved photoemission electron microscopy operated at sample illumination from the rear side

Review of Scientific Instruments 90, 053704 (2019); https://doi.org/10.1063/1.5088031

Absolute calibration of GafChromic film for very high flux laser driven ion beams

Review of Scientific Instruments 90, 053301 (2019); https://doi.org/10.1063/1.5086822

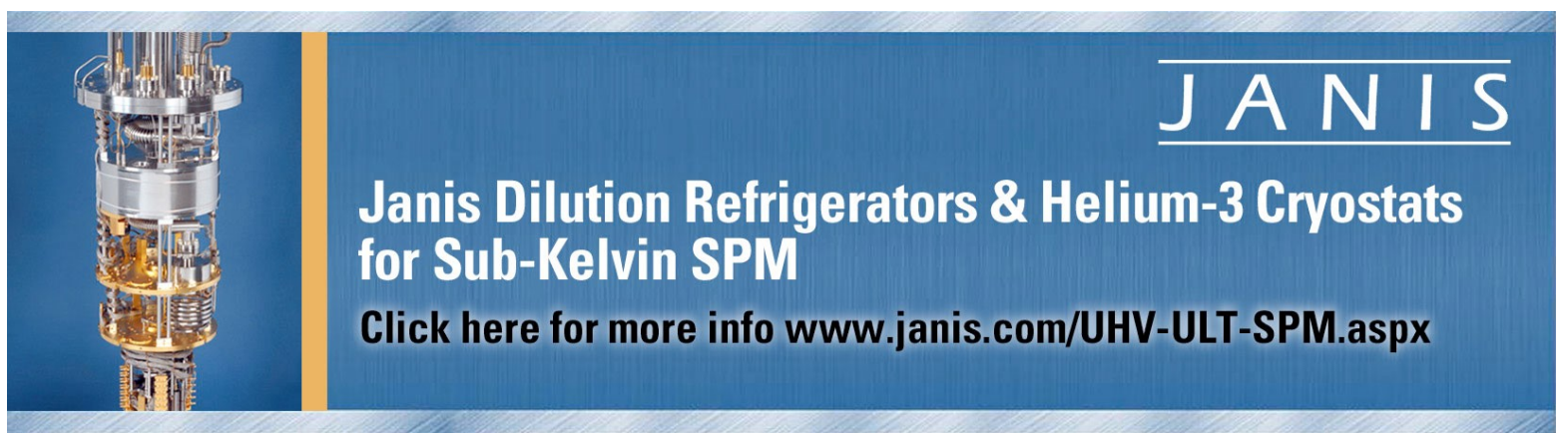




\title{
Rapid mixing of colliding picoliter liquid droplets delivered through-space from piezoelectric-actuated pipettes characterized by time-resolved fluorescence monitoring
}

\author{
Cite as: Rev. Sci. Instrum. 90, 055109 (2019); doi: 10.1063/1.5050270 \\ Submitted: 29 July 2018 - Accepted: 27 April 2019 • \\ Published Online: 21 May 2019
}

Jamie L. Y. Wu (巫柳依),

and R. J. Dwayne Miller ${ }^{2}$

Friedjof Tellkamp, ${ }^{2}$

Mazdak Khajehpour, ${ }^{3}$

Wesley D. Robertson,

\author{
AFFILIATIONS \\ ${ }^{1}$ Division of Engineering Science, University of Toronto, Toronto, Ontario M5S 2E4, Canada \\ ${ }^{2}$ Max Planck Institute for the Structure and Dynamics of Matter, Hamburg 27761, Germany \\ ${ }^{3}$ Department of Chemistry, University of Manitoba, Winnipeg, Manitoba R3T 2N2, Canada \\ ${ }^{4}$ Quantum Systems Division, Georgia Tech Research Institute, Atlanta, Georgia 30318, USA \\ ${ }^{5}$ Departments of Chemistry and Physics, University of Toronto, Toronto, Ontario M5S 3H6, Canada \\ a) Contributions: J. L. Y. Wu and W. D. Robertson contributed equally to this work. \\ b) Author to whom correspondence should be addressed: dwayne.miller@mpsd.mpg.de
}

\begin{abstract}
Rapid mixing of aqueous solutions is a crucial first step to study the kinetics of fast biochemical reactions with high temporal resolution. Remarkable progress toward this goal has been made through the development of advanced stopped-flow mixing techniques resulting in reduced dead times, and thereby extending reaction monitoring capabilities to numerous biochemical systems. Concurrently, piezoelectric actuators for through-space liquid droplet sample delivery have also been applied in several experimental systems, providing discrete picoliter sample volume delivery and precision sample deposition onto a surface, free of confinement within microfluidic devices, tubing, or other physical constraints. Here, we characterize the inertial mixing kinetics of two aqueous droplets ( $130 \mathrm{pl})$ produced by piezoelectricactuated pipettes, following droplet collision in free space and deposition on a surface in a proof of principle experiment. A time-resolved fluorescence system was developed to monitor the mixing and fluorescence quenching of 5-carboxytetramethylrhodamine (5-Tamra) and $N$ Bromosuccinimide, which we show to occur in less than $10 \mathrm{~ms}$. In this respect, this methodology is unique in that it offers millisecond mixing capabilities for very small quantities of discrete sample volumes. Furthermore, the use of discrete droplets for sample delivery and mixing in free space provides potential advantages, including the elimination of the requirement for a physical construction as with microfluidic systems, and thereby makes possible and extends the experimental capabilities of many systems.
\end{abstract}

Published under license by AIP Publishing. https://doi.org/10.1063/1.5050270

\section{INTRODUCTION}

Dynamic processes in biophysical phenomena occur over a huge time scale range that spans nanoseconds to seconds. ${ }^{1}$ Those occurring on the micro to millisecond regime are of particular importance as this time scale encompasses processes such as domain movement, loop reorientation, local folding/unfolding, and enzyme turnover. ${ }^{2,3}$ Therefore, understanding the relationship between protein dynamics and functions requires detailed study of the motions that occur in this time window. Conventional spectrometers typically cannot be used for reactions with lifetimes less than 10-20 s due to the dead time resulting from manual mixing and instrument 
limitations. ${ }^{4}$ The initial work of Chance clearly demonstrated that a convenient way to access aqueous reactions with millisecond lifetimes is through rapid mixing techniques. ${ }^{5}$ Since then, rapid mixing of aqueous solutions is often required to study the kinetics of fast chemical or biochemical reactions such as enzymatic processes and protein folding. ${ }^{5-7}$ Every rapid mixing technique must therefore contend with the following two challenges: minimizing both the mixing time and the amount of material consumed. State-of-the-art stopped-flow, continuous-flow, and laminar flow devices have successfully achieved rapid mixing, reducing dead times down to the millisecond ${ }^{8,9}$ and even down to the microsecond time scale. ${ }^{6,10,11}$ These approaches can coarsely be categorized as "active," requiring the input of external energy, or "passive," taking advantage of the inherent hydrodynamic properties of fluid flow to achieve mixing. ${ }^{12}$ External perturbations in active mixing can come in the form of mechanical, ${ }^{13}$ electrical, ${ }^{14,15}$ magnetic, ${ }^{16}$ acoustic, ${ }^{17}$ and thermal ${ }^{18}$ agitations, while passive mixing is often accomplished by exploiting phenomena such as hydrodynamic focusing ${ }^{19}$ as well as chaotic advection. ${ }^{2}$

The generation and manipulation of microdroplets or nanodroplets have also demonstrated considerable potential for rapidly mixing sample volumes in the picoliter to nanoliter regime. The viscous dissipation of inertia within the coalesced droplets is the primary mechanism for mixing, where the direction of molecular diffusion and fluid advection are constantly reorganized, thereby shortening diffusion length scales and increasing the mixing rate. These approaches range from using electrospray emitters for mass spectrometer monitoring of fast reactions where the mixing of nanodroplets was estimated to be complete within a few microseconds, ${ }^{21}$ to pressure-driven microfluidic platforms where $25 \mu \mathrm{m}$ diameter microdroplets have been shown to mix in winding microchannels in approximately $2 \mathrm{~ms}^{22}$ Carrol and Hidrovo described a mixer which produced fast colliding droplets confined within microchannels using high-speed gaseous flow, where the rapid mixing of aqueous droplets containing different concentrations of the fluorophore pyrromethene 556 occurred within $0.6 \mathrm{~ms}$ for high Reynolds number droplets. ${ }^{23}$ The tremendous advancements in these rapid droplet mixing devices enabled their use in conjunction with X-ray scattering techniques to probe the dynamics in macromolecular structures and biochemical reactions. Microfocus beamlines from synchrotron radiation (SR) and X-ray free electron lasers (XFEL) can be used in applications such as protein crystallography for determining the structure and dynamics of biological macromolecules. ${ }^{24,25}$ Recent developments in drop-on-demand (DOD) systems have been used to deposit droplets containing crystals of the enzyme thermolysin by acoustic droplet ejection (ADE) technology on to synchrotron X-ray-transparent substrates. ${ }^{26}$ A similar DOD system was also implemented with an XFEL source, providing time-resolved dynamics of the protein photosystem II. ${ }^{27}$ A study utilizing $80 \mu \mathrm{m}$ diameter ballistic microdroplets generated using a piezoelectric DOD device characterized the homogeneity of the protein analyte distribution following droplet collision. The experiment revealed an inhomogeneous analyte distribution at early times $(\sim 2 \mathrm{~ms})$, the onset of coalescence $(\sim 4 \mathrm{~ms})$ and diffusion and mixing beginning at longer times $(\sim 6 \mathrm{~ms}){ }^{28}$ Novel DOD injection systems have been developed and characterized using stroboscopic SR microbeam scattering techniques to probe single droplets of molten $\operatorname{wax}^{29}$ and aqueous solutions of cytochrome C. ${ }^{28,30}$ Droplet delivery systems have as well been utilized for the hydration of solid biopolymer samples subsequently examined using $x$-ray scattering. The surface energy of sufficiently small droplets (water $<100 \mu \mathrm{m}$ in diameter) dominates the kinetic energy and therefore the droplets impact a solid substrate without splashing, enabling precise spatial and temporal control over initiation of liquid-solid reactions. ${ }^{31,32}$ The ability to maintain coalescence of colliding droplets produced by a DOD system has demonstrated the method as a viable solution for mixing and injection into x-ray beamlines as well as numerous other experimental systems. ${ }^{33}$ However, for high intensity SR/XFEL microbeams, care must be taken to limit the effect of liquid explosive vaporization of DOD inkjet water droplets on serial crystallography experiments. The droplet must clear the probed volume between sampling events to avoid this problem and this limits the repetition rate of the signal acquisition. This effect can be used to advantage by using laser explosions to trigger pressure-driven molecular dynamics in solutions. $^{34}$

Given the efficient mixing of microdroplets reported in microfluidic systems and otherwise, the potential to deliver and mix sample or substrate via an individual microdroplet, through space and onto a surface, is applicable in numerous experimental scenarios. These include experiments where the sample volume is limited, as is the case with numerous proteomic applications ${ }^{35}$ or where there are significant experimental constraints, as in the case of serial crystallography, ${ }^{36}$ where the technique could provide a solution for fast, time resolved methods to probe the ligand binding induced structural dynamics contributions to enzymatic functions. Mixing in microdroplets has the potential to minimize sample consumption, and as a passive mixing methodology, it is suitable for applications involving sensitive species (i.e., biological samples) due to its lack of violent agitations. ${ }^{12}$ Here, we develop a system to deliver and characterize the inertial mixing of a pair of colliding and coalescing picoliter aqueous droplets produced by piezoelectric-actuated pipettes. The individual droplets travel through free space at highspeed $(2 \mathrm{~m} / \mathrm{s})$ and high-angle $\left(65^{\circ}\right)$ before their collision and concurrent deposition onto a glass slide. A time-resolved fluorescence monitoring setup was implemented to examine the mixing kinetics between a fluorescent probe and a fluorescence quencher. The delivery and mixing method requires minimal sample volume to operate; for example, when synchronized with $\mathrm{x}$-ray pulses, this technique could drastically reduce sample utilization in current nanocrystallography methods which are largely jet stream-based, consuming excessive amounts of sample. ${ }^{25,37}$ In addition, the contact-free delivery of single, unmixed, aqueous sample droplets onto a surface has numerous applications, including standard serial crystallography experiments. However, the droplet production frequency of DOD systems is unlikely to approach the XFEL repetition rates now available for which laser-based injector methods for high efficiency serial crystallography have been proposed. ${ }^{38}$ The method described here has the advantage of a simple system to deliver and mix discrete volumes of reagents such as biomolecules, substrates, and ligands separately and without the use of a continuous flow or microfluidic mixing device and could easily be employed for sample surface deposition and mixing in numerous time-resolved studies including SR or XFELs beamline interrogation of high-resolution time-resolved dynamics of biological macromolecules. 


\section{MATERIALS AND METHODS}

\section{A. Reagents}

5-carboxytetramethylrhodamine (5-Tamra) and $\mathrm{N}$ Bromosuccinimide (NBS) were purchased from Sigma-Aldrich (St. Louis, MO). All solutions were prepared in ultrapure water, degassed manually in a syringe, and micropore filtered (pore size $0.2 \mu \mathrm{m}$ ) before being loaded into the piezoelectric-actuated pipettes to ensure stable droplet generation and avoid clogging.

\section{B. Droplet generation}

Picoliter-volume aqueous droplets were generated and synchronized in free space using two commercially available piezoelectric-actuated pipettes (Autodrop Pipette AD-KH-501-L6) with their control electronics (MD-E-3020) from Microdrop Technologies $\mathrm{GmbH}$, Hamburg, Germany. In each pipette, the reagent solution was delivered into a glass capillary with an inner diameter of $70 \mu \mathrm{m}$ at the dispensing tip. The parameters for stable droplet production were optimized, and the droplet diameter was controlled by adjusting the pulse width of the voltage applied to the piezoelectric crystal. Droplet diameters of $60 \mu \mathrm{m}$ were used in all experiments, which resulted in a volume of $130 \mathrm{pl}$ for each individual droplet. The droplet velocity at the exit of the dispensing tip of the piezoelectric-actuated pipettes was approximately $2 \mathrm{~m} / \mathrm{s}$. Two discrete droplets were produced containing either the fluorophore 5-Tamra or NBS, which was used to quench 5-Tamra fluorescence in the experiment. Here, individual droplets with an approximate Reynolds number of 127 were used, though the setup is capable of producing water droplets at $22{ }^{\circ} \mathrm{C}$ with Reynolds numbers ranging from 71 to 148 .

The droplets were imaged in free space using a monochrome CCD camera. Figure 1(a) shows a representative image of the droplets after leaving the piezoelectric-actuated pipettes. The frequency of a strobe light connected to the pipette control electronics was synchronized to the rate at which droplets were dispensed from the pipettes, with an adjustable delay, such that the camera was able to capture the droplets trajectory through space. A long working distance microscope ( $95 \mathrm{~mm}$, QIOPTIQ, Waltham, USA) was used to image the droplet impact perpendicular to the plane of droplet trajectory and utilized additionally to perform alignment. The position and angles of the pipettes were adjusted such that the droplet of aqueous 5-Tamra solution and the droplet of aqueous NBS solution
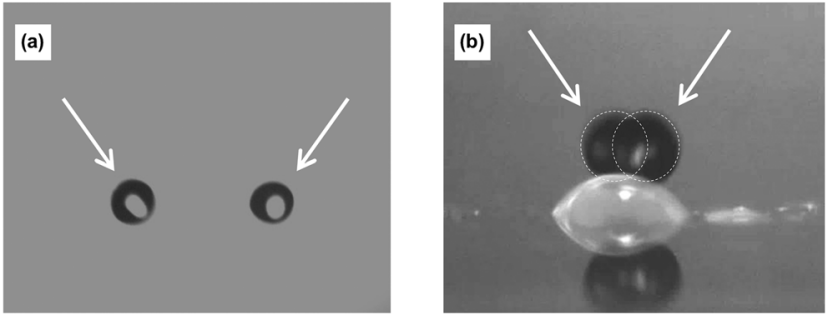

FIG. 1. (a) Droplets (60 $\mu \mathrm{m}$ in diameter; $130 \mathrm{pl})$ dispensed from two piezoelectricactuated pipettes in flight, as captured by the CCD camera. (b) Two droplets coalescing before impact on the glass slide. Here, the sample slide has not been scanned and a droplet is visible on the slide from the previous droplet impact. collided, coalesced, and mixed as they impacted a microscope slide as shown in Fig. 1(b). The total path length through space for each droplet before impact was approximately $3.5 \mathrm{~mm}$. The mixing and impact occurred within $2 \mathrm{~ms}$ of the droplets leaving their respective pipettes.

\section{Time-resolved fluorescence monitoring setup}

A schematic illustration of the mechanical and optical, timeresolved fluorescence monitoring setup is shown in Fig. 2. The fluorescence excitation source was provided by a green Helium Neon (HeNe) laser (HGP005; $543 \mathrm{~nm}, 0.5 \mathrm{~mW}$ ). An oscilloscope (Agilent InfiniiVision) was connected to the output of a photomultiplier tube (PMT, Hamamatsu R928) to monitor the fluorescence signal from the coalesced droplet. The PMT was operated at $1000 \mathrm{~V}$ provided by a power supply module (C12446-12) from Hamamatsu. The oscilloscope was terminated with a resistance of $70 \mathrm{k} \Omega$ resulting in a time constant of $5 \mu \mathrm{s}$. A dichroic filter (MDF72ITC), an excitation filters (MF542-20), an emission filter (MF620-52), a multimode optical fiber (105 $\mu \mathrm{m}$, M43L01), as well as other collimating optical elements were purchased from ThorLabs and used to deliver the excitation beam aimed at the coalesced droplet as well as to collect fluorescence signal emission. The system was enclosed to minimize stray light to the PMT. The excitation volume of the HeNe green laser and the collection volume of the emitted red fluorescence were approximately $200 \mu \mathrm{m}$ in diameter at the plane of focus of the microscope objective lens (RMS4X-4X).

\section{Experimental setup and data acquisition}

A pulse generator was used to trigger the control electronics of the piezoelectric-actuated pipettes individually in order to coordinate the timing of each droplet collision event to data acquisition. A home-designed and constructed positioning system was used to adjust the pipettes' angle and position. The parameters for droplet generation (pulse width, voltage, and pulse delay) were adjusted to facilitate droplet collision. The highly inertial condition of the traveling droplets and their collision on the glass surface facilitated rapid mixing. Coalescence of the droplets coincided with their deposition on the glass surface as shown in Fig. 1. During data collection, the frequency of droplet generation was set to $0.1 \mathrm{~Hz}$ with the pulse generator to facilitate fluorescent signal collection. A thin $(500 \mu \mathrm{m}$ thick) coverslip was placed on top of the microscope slide and translated horizontally after each droplet collision and deposition onto the surface. This provided a clean surface for the mixing event of each droplet pair.

The mixing time (dead time) of the system was measured by monitoring the reaction between 5-Tamra and a series of NBS concentrations following the coalescence of the droplets. This was done by measuring the fluorescence signal of 5-Tamra as a function of time. The concentration of the 5-Tamra fluorophore stock solution was kept constant at $11.6 \mu \mathrm{M}$ in all experiments. The concentration of NBS was held greater than 10-fold excess relative to the 5-Tamra in order to ensure pseudo first-order reaction kinetics. ${ }^{4,8}$ The final molar concentrations of 5-Tamra and NBS after mixing two equal-size droplets were therefore $5.8 \mu \mathrm{M}$ 


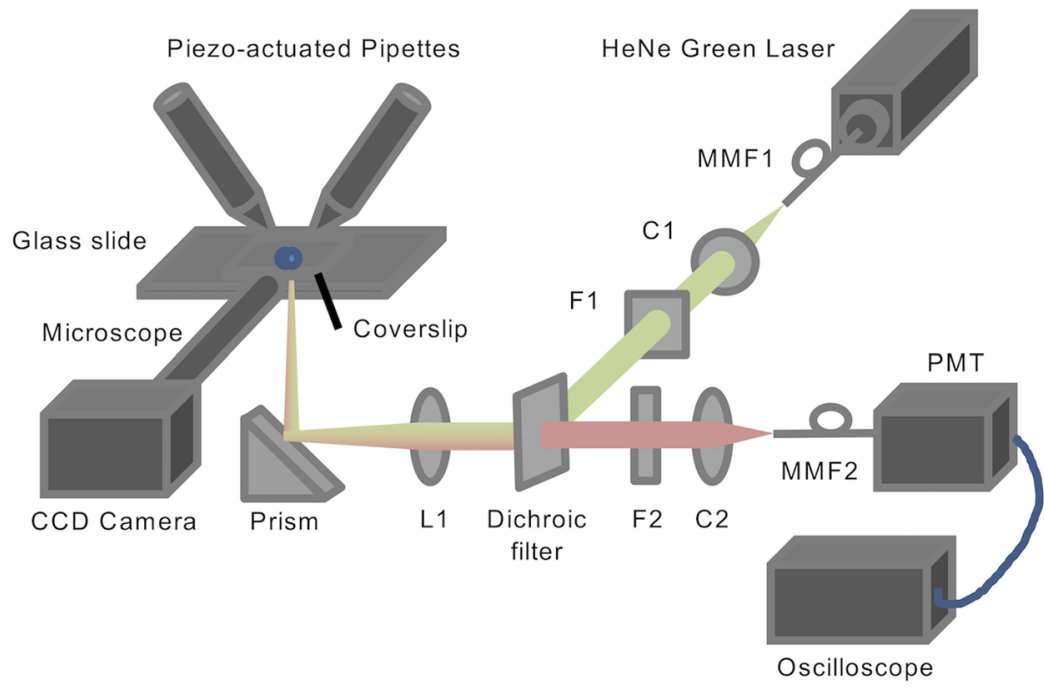

FIG. 2. Schematic illustration of the mechanical and optical setup of the time-resolved fluorescence monitoring system (L: lens, F: filter, C: collimator, and MMF: Multimode optica fiber).

and between $0.875 \mathrm{mM}$ and $14 \mathrm{mM}$, respectively. Each reaction profile shown in Fig. 3 is the average of at least 16 discrete droplet impact events, producing a single fluorescence decay trace. The experiments at each concentration were then performed in triplicate.

\section{E. Data processing and analysis}

Data from the time-resolved fluorescence signal trace were analyzed and presented using MATLAB. The three data sets were normalized at each quencher concentration through multiplying each data point by a gauge factor such that the medians of the last 10 data points of each graph coincide, following Bleul et al.'s method. ${ }^{8}$ The average of the normalized data sets was than

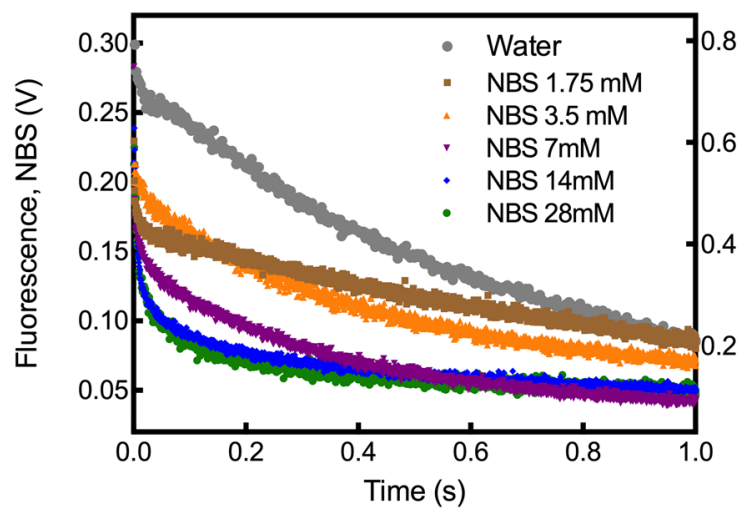

FIG. 3. Fluorescence signal decay traces of $11.6 \mu \mathrm{M} 5$-Tamra droplet following collision and mixing with an NBS droplet at concentrations ranging from $0 \mathrm{mM}$ to $28 \mathrm{mM}$. The control trace mixing $11.6 \mu \mathrm{M}$ 5-Tamra with water (0 mM NBS) is plotted in gray. The right-hand $y$-axis is associated with the decay in fluorescence observed in 5-Tamra in pure water, while the left-hand $y$-axis is associated with all the other decays. At higher NBS concentrations, fluorescence signal measured at time zero was lower, and the quenching reaction proceeded more rapidly. fitted to provide single time constants for each term in the model at each NBS concentration. The fit was carried out to $1 \mathrm{~s}$, and the data spacing was $625 \mu$ s and $2.5 \mathrm{~ms}$ for the control trace with water.

\section{RESULTS AND DISCUSSIONS}

The reaction of NBS with aromatic molecules is a rapid secondorder oxidative reaction that has often been used to characterize the dead time in rapid mixing experiments. ${ }^{39}$ The oxidation of 5-Tamra leads to a loss in fluorescence which can easily be monitored by our optical setup. The normalized and averaged reaction traces for each quencher concentration are shown in Fig. 3. It can be observed that increasing the amount of NBS causes an increase in the rate of 5-Tamra oxidation as well as lowering the value of fluorescence measured at time zero. This indicates that at high concentrations of NBS, the oxidative reaction rate exceeds the detection limits of our instrumental setup. Further examination of the reaction profiles in Fig. 3 demonstrate that the data can be readily fit to a multiexponential function of the form: Fluorescence $(t)=\Sigma a_{i} e^{-k_{i} t}$. A nonlinear least-square method was implemented in MATLAB for curve fitting. By examining the goodness-of-fit statistics, residual plots, and fit parameter confidence bounds, it was determined that the reaction profiles at times less than one second were well-described by a biexponential decay plus a constant, with all fits generating R-square values above 0.99 , where $a_{1}, k_{1}, a_{2}, k_{2}$, and $c$ are the fit parameters

$$
\text { Fluorescence }(t)=a_{1} e^{-k_{1} t}+a_{2} e^{-k_{2} t}+c \text {. }
$$

The control trial without NBS present in the colliding water droplet was recorded and fit to a signal exponential decay plus a constant term. Values of the fitted parameters obtained are tabulated in Table I. It is evident that the slower apparent rate constant, $k_{2}$, shows no appreciable dependence on the concentration of NBS, while the faster rate constant, $k_{1}$, markedly depends upon the concentration of NBS. These dependencies have been plotted in Fig. 4. 
TABLE I. Tabulated values of $k_{1}$, the rate constant associated with the fast decay component and which is NBS concentration dependent; $k_{2}$, the rate constant associated with the slow decay component and which remained relatively consistent across NBS concentrations tested, and; $c$, a constant to fit long time scale background signal. Values for the coefficients associated with each exponential decay term, $a_{1}$ and $a_{2}$, are also shown. Here, the bracketed values represent the $95 \%$ confidence intervals (lower bound, upper bound) for the fitted values. The rate constants associated with each exponential decay term are shown in bold font and plotted in Fig. 4.

\begin{tabular}{|c|c|c|c|c|c|}
\hline NBS conc. (mM) & $a_{1}(\mathrm{~V})$ & $k_{1}\left(\mathrm{~s}^{-1}\right)$ & $a_{2}(\mathrm{~V})$ & $k_{2}\left(\mathrm{~s}^{-1}\right)$ & $c(\mathrm{~V})$ \\
\hline 0 (water) & & & $0.644(0.643,0.646)$ & $1.55(1.54,1.56)$ & $0.198(0.196,0.199)$ \\
\hline 1.75 & $0.018(0.017,0.019)$ & $36.44(34.69,38.19)$ & $0.142(0.141,0.144)$ & $3.87(3.74,3.99)$ & $0.039(0.038,0.039)$ \\
\hline 3.5 & $0.028(0.028,0.028)$ & $\mathbf{5 6 . 8 3}(55.69,57.98)$ & $0.158(0.157,0.158)$ & $2.02(2.01,2.03)$ & $0.035(0.034,0.035)$ \\
\hline 7 & $0.039(0.039,0.039)$ & $76.93(76.04,77.81)$ & $0.105(0.105,0.105)$ & $2.85(2.84,2.86)$ & $0.036(0.036,0.036)$ \\
\hline 14 & $0.099(0.098,0.099)$ & $100.90(100.30,101.50)$ & $0.055(0.055,0.055)$ & $3.30(3.28,3.33)$ & $0.050(0.050,0.050)$ \\
\hline 28 & $0.056(0.056,0.057)$ & $115.60(114.60,116.70)$ & $0.122(0.121,0.122)$ & $4.50(4.38,4.61)$ & $0.051(0.051,0.051)$ \\
\hline
\end{tabular}

As previously discussed, the reaction profile of 5-Tamra and NBS, as characterized by their fluorescence exhibits, two decay phases, one fast and the other slow. The magnitude of the rate constants $k_{2}$ associated with the slow decay are more than 10 times slower than that of $k_{1}$, an indication that these two processes are not coupled with each other. One important consideration that must be made is that the experimental setup is not mechanically enclosed and is exposed to atmosphere, and therefore droplet evaporation must also be taken into account. Given the fact that the value of $k_{2}$ is independent of NBS concentration, it is likely that the decay associated with this parameter is due to evaporation and subsequent fluorescence self-quenching, bleaching, and interfilter effects resulting from increased fluorophore concentration as the droplet volume decreases. ${ }^{40}$ Changes in the shape or complexities in the fluid dynamics after initial collision of the droplets onto the glass surface likely contributes to the decay as well. Our hypothesis is strengthened by the fact that the same decay is observed when there is no NBS present and 5-Tamra is just mixed with water, as shown in Fig. 3. This control data was fit well with a longer time scale single exponential term $\left(k_{2}=1.55 \mathrm{~s}^{-1}\right)$ plus a constant

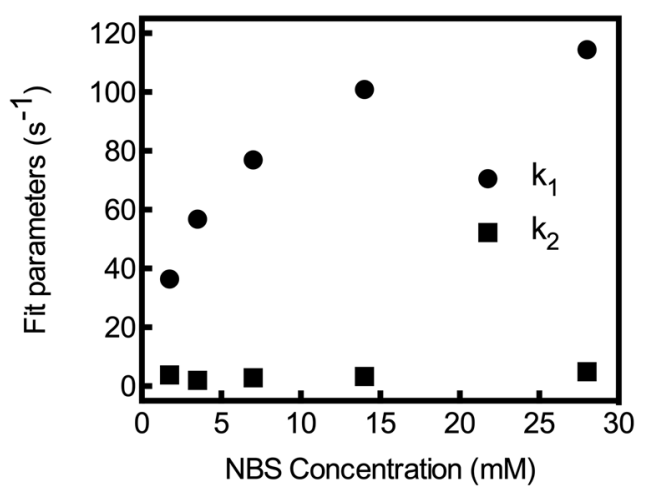

FIG. 4. The first-order rate constant, $k_{1}$, which characterizes the mixing and quenching reaction kinetics, is determined from the curve fitting for each fluorescence signal trace at a given NBS concentration. As NBS concentration increases, the overall reaction rate becomes limited by the mixing rate of the two droplets, therefore the reaction rate plateaus and becomes independent of the NBS concentration. The rate constant, $k_{2}$, attributed to droplet evaporation, remains consistent and is independent of NBS concentrations.
( $c=0.198)$. Exponential decay-based models with the same time scale have been also been used in the past to describe the evaporation of atmospherically exposed solution droplets. ${ }^{41,42}$ More advanced models for the evaporation of surface deposited droplets containing colloidal or nanoparticle suspensions ${ }^{43}$ have been developed and more accurately describe this process. Additionally, a model for the mixing and reaction competition occurring during droplet coalescence has been revealed by scanning small-angle $\mathrm{x}$-ray scattering (SAXS) measurements. ${ }^{28}$ The combination of these approaches should provide the basis for more exact modeling of mixing, reaction, and evaporation following droplet collision. In the case of this set of experiments however, it is clear that the evaporation process is much slower than the mixing and reaction processes, and therefore, we may assume that the evaporation kinetics is decoupled from that of mixing and reaction. This assumption significantly simplifies our kinetic model.

As observed in Fig. 4, the rate constants associated with the fast decay $k_{1}$ show significant dependence on NBS concentration: at low NBS concentrations, $k_{1}$ increases rapidly as the concentration is increased while at high concentrations $k_{1}$ approaches a plateau. This is not consistent with a simple second order reaction, indicating a multistep process. The simplest model we can propose that is consistent with this result is the following scheme:

$$
\begin{gathered}
\text { drop } \text { containing } 5-\text { Tamra }+ \text { drop }_{\text {containing NBS }} \\
\stackrel{k_{\text {mixing }}}{\longrightarrow} \text { large drop containing 5-Tamra and NBS } \\
\text { large drop containing 5-Tamra and NBS } \stackrel{k_{\text {reaction }}}{\longrightarrow} \text { oxidized 5 - Tamra. }
\end{gathered}
$$

The concentration dependences observed in $k_{1}$ can be explained as the following. At low concentrations of NBS, the rate of mixing is faster than that of reaction. Given that the concentration of NBS is in excess of 5-Tamra, the reaction should follow pseudo-first order kinetics and the observed rate constant should show a linear dependence on NBS concentration. As the NBS concentration increases, the reaction rate becomes faster and the reaction rate is no longer limited by the chemistry but instead becomes limited by the rate in which the two droplets mix with each other. This rate is independent of the concentration of NBS. The sum of these two processes would therefore lead to the rise and the consequent plateau observed in the $\mathrm{k}_{1}$ values depicted in Fig. 4 . In this case, the limit of $k_{1}$ at high 
concentrations of NBS should be larger than $115.6 \mathrm{~s}^{-1}$. This rate corresponds to an upper limit of $9 \mathrm{~ms}$ for the mixing time, which is faster than the reaction time of many biochemical processes.

\section{CONCLUSIONS}

The through-space delivery and rapid mixing of picoliter droplets using piezoelectric-actuated pipettes has been presented here in a proof of principle experiment as a method for sample delivery and for the investigation of fast chemical or biochemical reaction schemes. Taking advantage of the system's time-resolved fluorescence monitoring capability, recording and analysis of the quenching reaction between the fluorophore 5-Tamra and quencher NBS found the system mixing time to be less than $9 \mathrm{~ms}$. The platform is adaptable to deliver, mix, and deposit small volumes of substrates, ligands, and other biomolecules tagged with fluorescence probes to investigate their time-resolved interaction. The technique provides experimental flexibility in delivering and mixing chemical and biological reagents and a handle to further study their reaction kinetics using a variety of monitoring techniques, while requiring a low sample volume and no microfluidic restrictions. Further studies will focus on optimizing the mixing time of the colliding droplets by adjusting the droplet size and velocity as well as characterization of mixing rates for different Reynolds number droplets. There are particularly important applications of this approach for structural studies of enzyme reaction mechanisms using $\mathrm{x}$-ray structural probes in recently developed microfocus beamlines.

\section{ACKNOWLEDGMENTS}

This work was supported by the Max Planck Society. We would like to thank Jessica Besaw and William Stuart for their help in the assembly and testing of the Microdrop autopipette system.

\section{REFERENCES}

${ }^{1}$ R. Callender and R. B. Dyer, Chem. Rev. 106, 3031 (2006).

${ }^{2}$ K. A. Henzler-Wildman, M. Lei, V. Thai, S. J. Kerns, M. Karplus, and D. Kern, Nature 450, 913 (2007).

${ }^{3}$ A. Mittermaier and L. E. Kay, Science 312, 224 (2006)

${ }^{4}$ S. R. Martin and M. J. Schilstra, Protein-Ligand Interactions: Methods and Applications (Springer Science Business Media, New York, 2013), pp. 119-138.

${ }^{5}$ B. Chance, J. Biol. Chem. 151, 553 (1943), ISSN 0021-9258.

${ }^{6}$ S. Vahidi, B. B. Stocks, Y. Liaghati-Mobarhan, and L. Konermann, Anal. Chem. 85, 8618 (2013).

${ }^{7}$ S. Akiyama, S. Takahashi, T. Kimura, K. Ishimori, I. Morishima, Y. Nishikawa, and T. Fujisawa, Proc. Natl. Acad. Sci. U. S. A. 99, 1329 (2002).

${ }^{8}$ R. Bleul, M. Ritzi-Lehnert, J. Höth, N. Scharpfenecker, I. Frese, D. Düchs, S. Brunklaus, T. E. Hansen-Hagge, F.-J. Meyer-Almes, and K. S. Drese, Anal. Bioanal. Chem. 399, 1117 (2011)

${ }^{9}$ M. Guo, B. Bhaskar, H. Li, T. P. Barrows, and T. L. Poulos, Proc. Natl. Acad. Sci. U. S. A. 101, 5940 (2004).

${ }^{10}$ A. V. Cherepanov and S. De Vries, Biochim. Biophys. Acta 1656, 1 (2004).

${ }^{11}$ M. C. Shastry, S. D. Luck, and H. Roder, Biophys. J. 74, 2714 (1998).

${ }^{12}$ A. P. Sudarsan and V. M. Ugaz, Proc. Natl. Acad. Sci. U. S. A. 103, 7228 (2006).

${ }^{13}$ B. A. Cola, D. K. Schaffer, T. S. Fisher, and M. A. Stremler, J. Microelectromech. Syst. 15, 259 (2006).

${ }^{14}$ Y.-K. Lee, J. Deval, P. Tabeling, and C.-M. Ho, in The 14th IEEE Workshop in $M E M S$ (IEEE, 2001).
${ }^{15}$ M. H. Oddy, J. G. Santiago, and J. C. Mikkelsen, Anal. Chem. 73, 5822 (2001).

${ }^{16}$ H. H. Bau, J. Zhong, and M. Yi, Sens. Actuators B 79, 207 (2001).

${ }^{17}$ D. Ahmed, X. Mao, J. Shi, B. K. Juluri, and T. J. Huang, Lab Chip 9, 2738 (2009)

${ }^{18}$ A. N. Hellman, K. R. Rau, H. H. Yoon, S. Bae, J. F. Palmer, K. S. Phillips, N. L. Allbritton, and V. Venugopalan, Anal. Chem. 79, 4484 (2007).

${ }^{19}$ D. Wang, U. Weierstall, L. Pollack, and J. Spence, J. Synchrotron Radiat. 21, 1364 (2014).

${ }^{20}$ H. Aref, J. Fluid Mech. 143, 1 (1984).

${ }^{21}$ D. N. Mortensen and E. R. Williams, Anal. Chem. 86, 9315 (2014).

${ }^{22}$ H. Song, D. L. Chen, and R. F. Ismagilov, Angew. Chem., Int. Ed. 45, 7336 (2006).

${ }^{23}$ B. Carroll and C. Hidrovo, Heat Transfer Eng. 34, 120 (2013).

${ }^{24}$ M. Yamamoto, K. Hirata, K. Yamashita, K. Hasegawa, G. Ueno, H. Ago, and T. Kumasaka, IUCrJ 4, 529 (2017).

${ }^{25}$ H. N. Chapman, P. Fromme, A. Barty, T. A. White, R. A. Kirian, A. Aquila, M. S. Hunter, J. Schulz, D. P. DePonte, U. Weierstall, R. B. Doak, F. R. N. C. Maia, A. V. Martin, I. Schlichting, L. Lomb, N. Coppola, R. L. Shoeman, S. W. Epp, R. Hartmann, D. Rolles, A. Rudenko, L. Foucar, N. Kimmel, G. Weidenspointner, P. Holl, M. Liang, M. Barthelmess, C. Caleman, S. Boutet, M. J. Bogan, J. Krzywinski, C. Bostedt, S. Bajt, L. Gumprecht, B. Rudek, B. Erk, C. Schmidt, A. Hömke, C. Reich, D. Pietschner, L. Strüder, G. Hauser, H. Gorke, J. Ullrich, S. Herrmann, G. Schaller, F. Schopper, H. Soltau, K.-U. Kühnel, M. Messerschmidt, J. D. Bozek, S. P. Hau-Riege, M. Frank, C. Y. Hampton, R. G. Sierra, D. Starodub, G. J. Williams, J. Hajdu, N. Timneanu, M. M. Seibert, J. Andreasson, A. Rocker, O. Jönsson, M. Svenda, S. Stern, K. Nass, R. Andritschke, C.-D. Schröter, F. Krasniqi, M. Bott, K. E. Schmidt, X. Wang, I. Grotjohann, J. M. Holton, T. R. M. Barends, R. Neutze, S. Marchesini, R. Fromme, S. Schorb, D. Rupp, M. Adolph, T. Gorkhover, I. Andersson, H. Hirsemann, G. Potdevin, H. Graafsma, B. Nilsson, and J. C. H. Spence, Nature 470, 73 (2011).

${ }^{26}$ C. G. Roessler, A. Kuczewski, R. Stearns, R. Ellson, J. Olechno, A. M. Orville, M. Allaire, A. S. Soares, and A. Héroux, J. Synchrotron Radiat. 20, 805 (2013).

${ }^{27}$ F. D. Fuller, S. Gul, R. Chatterjee, E. S. Burgie, I. D. Young, H. Lebrette, V. Srinivas, A. S. Brewster, T. Michels-Clark, J. A. Clinger, B. Andi, M. Ibrahim, E. Pastor, C. de Lichtenberg, R. Hussein, C. J. Pollock, M. Zhang, C. A. Stan, T. Kroll, T. Fransson, C. Weninger, M. Kubin, P. Aller, L. Lassalle, P. Bräuer, M. D. Miller, M. Amin, S. Koroidov, C. G. Roessler, M. Allaire, R. G. Sierra, P. T. Docker, J. M. Glownia, S. Nelson, J. E. Koglin, D. Zhu, M. Chollet, S. Song, H. Lemke, M. Liang, D. Sokaras, R. Alonso-Mori, A. Zouni, J. Messinger, U. Bergmann, A. K. Boal, J. M. Bollinger, Jr., C. Krebs, M. Högbom, G. N. Phillips, Jr., R. D. Vierstra, N. K. Sauter, A. M. Orville, J. Kern, V. K. Yachandra, and J. Yano, Nat. Methods 14, 443 (2017).

${ }^{28}$ R. Graceffa, M. Burghammer, R. J. Davies, and C. Riekel, Appl. Phys. Lett. 101, 254101 (2012).

${ }^{29}$ R. Graceffa, M. Burghammer, R. J. Davies, and C. Riekel, Rev. Sci. Instrum. 79, 086106 (2008).

${ }^{30}$ R. Graceffa, M. Burghammer, R. J. Davies, C. Ponchut, and C. Riekel, Appl Phys. Lett. 94, 062902 (2009).

${ }^{31}$ M. Rössle, D. Flot, J. Engel, M. Burghammer, C. Riekel, and H. Chanzy, Biomacromolecules 4, 981 (2003).

${ }^{32}$ C. Riekel, M. Burghammer, D. Flot, and M. Rössle, Fibre Diffr. Rev. 12, 36 (2004).

${ }^{33}$ R. Graceffa, Development of a Drop-On-Demand Inkjet System for Stroboscopic Small-and Wide-Angle X-Ray Scattering Experiments (Joseph Fourier University, Grenoble, France, 2010).

${ }^{34}$ C. A. Stan, D. Milathianaki, H. Laksmono, R. G. Sierra, T. A. McQueen, M. Messerschmidt, G. J. Williams, J. E. Koglin, T. J. Lane, M. J. Hayes, S. A. H. Guillet, M. Liang, A. L. Aquila, P. R. Willmott, J. S. Robinson, K. L. Gumerlock, S. Botha, K. Nass, I. Schlichting, R. L. Shoeman, H. A. Stone, and S. Boutet, Nat. Phys. 12, 966 (2016).

${ }^{35}$ M. J. Jebrail, V. N. Luk, S. C. C. Shih, R. Fobel, A. H. C. Ng, H. Yang, S. L. S. Freire, and A. R. Wheeler, J. Vis. Exp. 33, e1603 (2009).

${ }^{36}$ M. Heymann, A. Opthalage, J. L. Wierman, S. Akella, D. M. E. Szebenyi, S. M. Gruner, and S. Fraden, IUCrJ 1, 349 (2014).

${ }^{37}$ M. O. Wiedorn, S. Awel, A. J. Morgan, K. Ayyer, Y. Gevorkov, H. Fleckenstein, N. Roth, L. Adriano, R. Bean, K. R. Beyerlein, J. Chen, J. Coe, F. Cruz-Mazo, 
T. Ekeberg, R. Graceffa, M. Heymann, D. A. Horke, J. Knoška, V. Mariani, R. Nazari, D. Oberthür, A. K. Samanta, R. G. Sierra, C. A. Stan, O. Yefanov, D. Rompotis, J. Correa, B. Erk, R. Treusch, J. Schulz, B. G. Hogue, A. M. Gañán-Calvo, P. Fromme, J. Küpper, A. V. Rode, S. Bajt, R. A. Kirian, and H. N. Chapman, IUCrJ 5, 574 (2018)

${ }^{38}$ E. C. Schulz, J. Kaub, F. Busse, P. Mehrabi, H. M. Müller-Werkmeister, E. F. Pai, W. D. Robertson, and R. J. Dwayne Miller, J. Appl. Crystallogr. 50, 1773 (2017).
${ }^{39}$ B. F. Peterman, Anal. Biochem. 93, 442 (1979).

${ }^{40}$ J. R. Lakowicz, Probe Design and Chemical Sensing, Topics in Fluorescence Spectroscopy (Springer Science \& Business Media, 2006), Vol. 4.

${ }^{41}$ A. A. Cantú, Forensic Sci. Int. 219, 119 (2012).

${ }^{42} \mathrm{H}$. J. Holterman, Kinetics and Evaporation of Water Drops in Air (IMAG, Wageningen, Netherlands, 2003).

${ }^{43}$ Y. O. Popov, Phys. Rev. E 71, 036313 (2005) 